\title{
Audit of Prescribing Practices to Evaluate Rational Use of Medicines in the OPD of Orthopaedics in a Private Medical College Hospital
}

\author{
Afsan $\mathrm{M}^{1}$, Haque $\mathrm{MME}^{2}$, Alam $\mathrm{MM}^{3}$, Noor $\mathrm{N}^{4}$
}

\begin{abstract}
Background: Prescribing patterns need to be evaluated periodically to increase the therapeutic efficacy, decrease adverse effects and provide feedback to prescribers. Like all other developing countries, irrational and inappropriate use of drugs is very common in Bangladesh. Objectives: The main objective of the prescription audit was to define the pattern of drug used in the outpatient department of orthopaedics. Methodology: This was a descriptive type of cross-sectional study. The study was conducted in the Outpatient Department (OPD) of Orthopaedics in a tertiary care private teaching hospital. Dhaka, Bangladesh in between January to March '2012. A total of 300 prescriptions were obtained with the help of a pre-inserted carbon paper in a special format using WHO core prescribing indicators and some additional indices. Results: The average number of drugs per encounter was 3.78 and no single drug was prescribed by generic name. Use of an antibiotic and an injection was in $6.67 \%$ and $3.33 \%$ of encounters respectively. Only $4.32 \%$ drugs were prescribed from national essential drug list (EDL). Percentage of encounters with a NSAID, an antiulcerant and a calcium preparation prescribed were $97 \%, 97.33 \%$ and $67.33 \%$ respectively. Conclusion: The patterns of drug prescribing in the OPD of orthopaedics did not fulfill the WHO criteria for rational use of medicine.
\end{abstract}

Key words: Prescribing practice, rational use of medicine, audit, drug prescription

\section{Introduction}

Drug utilization studies are pre requisite for the formulation of drug policies. These studies also offer useful methods for teaching and training in drug therapy. It is well known that indiscriminate use of drug results in unwanted adverse effects and drug interactions and poses difficulties in diagnosis. Manufacturers create artificial demand for unwanted drugs and drug combination through competitive sale promotions. The drug utilization study identifies the problems that arise from drug usage in healthcare delivery system and highlights the current approaches to the rational use of medicines ${ }^{1}$.

Drug utilization research has been defined by the World Health Organization (WHO) in 1977 as the marketing, distribution, prescription and use of drugs in a society, with special emphasis on the resulting medical, social and economic consequences ${ }^{2}$. Drug utilization review is defined as authorized, structured and continuing program that reviews analyses and interprets the pattern of drug use against pre-determined standards ${ }^{3}$. The conference of experts on the rational use of drugs, convened by the World
Health Organization (WHO) in Nairobi (Kenya) in 1985 defined that rational use of drugs requires that patients receive medications appropriate to their clinical needs, in doses that meet their own individual requirements, for an adequate period of time and at the lowest cost to them and their community ${ }^{2}$. Prescribers can only treat patients in a rational way if they have access to an essential medicines list and essential medicines are available on a regular basis ${ }^{4}$. The irrational and inappropriate use of drugs is a well documented universal problem and is a major concern for developing countries, as has been recognized by the World Health Organization (WHO $)^{5}$. Now-a-days the prescribing pattern is changing and it has become just an indication of medicine with some instructions of doses without considering its rationality ${ }^{6}$. The common irrational prescribing patterns include polypharmacy, drugs prescribed by trade names, inappropriate use of antimicrobials, unnecessary use of tonics and other non-essential drugs? Irrational drug use leads to reduction in the quality of drug therapy, wastage of resources, increased treatment cost, increased risk for adverse drug reactions and emergence of drug resistance ${ }^{8}$.

1. Dr. Mohammad Afsan, Associate Professor \& Head, Department of Pharmacology and Therapeutics,, East-West Medical College, Dhaka

2. Dr. M.M. Ekramul Haque, Assistant Professor, Department of Orthopaedics, East-West Medical College, Dhaka

3. Dr. Muhammad Mahbubul Alam, Assistant Professor, Department of Pharmacology and Therapeutics, East-West Medical College, Dhaka

4. Dr. Nushrat Noor, Assistant Professor, Department of Physiology, Dhaka Central International Medical College, Dhaka

\section{Correspondence}

Dr. Mohammad Afsan, Associate Professor and Head, Department of Pharmacology, East-West Medical College, P.O.Khayertek,Turag,Dhaka, Bangladesh; Email: mafsan@yahoo.com; Cell no.: +8801712152360 
The assessment of drug utilization is important for clinical, educational and economic purposes specially in a developing country like Bangladesh. It may also help the clinician to take appropriate measure for the improvement of prescribing patterns and to prevent prescribing errors and thus promote rational use of medicines. Prescribers can only treat patients in a rational way if they have access to an essential medicines list and essential medicines are available on a regular basis. Therefore, the present study has been undertaken to observe the prescribing patterns in a tertiary level private hospital in Dhaka. Inappropriate drug prescribing is a global problem. Keeping all these facts in consideration, the present study has been planned to define the pattern of drug use in a private medical college hospital of Dhaka. Other objectives were to articulate measures for improving the prescription practices and to generate information on the core prescribing indicators proposed by the World Health Organization (WHO).

\section{Methodology}

This descriptive type of cross-sectional study was conducted in East-West Medical College Hospital (EWMCH), Dhaka. The study was carried out over a 3 months period from January' 2012 to March'2012. The patients and their prescriptions were used as source of data. A total of 300 patients were included in this study. New patients attending the Outpatient Department of Orthopaedics which is located in the ground floor of East-West Medical College Hospital during the study period were considered for analysis. Follow up visits during the study period were included and were counted as separate visits. Patients visiting the emergency department or who got admitted during OPD visit were not included in the study. To assess the scope for improvement in rational drug use in outpatient practice, the World Health Organization (WHO) in 1993 has formulated a set of "Core drug use indicators" namely prescribing indicators, patient care indicators and facility indicators. Among them, for this study only "prescribing indicators" were taken which measure the performance of prescribers. The core prescribing indicators are average number of drugs per prescription, percentage of drugs prescribed by generic name, percentage of encounters with an antibiotic prescribed, percentage of encounters with an injection prescribed and percentage of drugs prescribed from essential drug list or formulary. Some additional indices are percentage of encounters with a NSAID prescribed, percentage of encounters with an antiulcerant prescribed, percentage of encounters with a calcium preparation prescribed and the data was expressed as percentage, mean and total numbers.

\section{Results}

A total of 1134 individual drugs were prescribed for 300 drug encounters, giving an average of 3.78. The range of drugs per encounter varied from 1-7. There was not a single prescription wherein no drug was prescribed. As shown in Table 1 four (4) drugs were prescribed in 158(52.7\%) and prescriptions was found to be highest among 300 prescriptions.

Table 1: Number of drugs prescribed per prescription

\begin{tabular}{lr}
\hline Prescription Containing & $\begin{array}{r}\text { Number of } \\
\text { Prescriptions }\end{array}$ \\
\hline One & $2(0.67)$ \\
Two & $10(3.33)$ \\
Three & $89(29.67)$ \\
Four & $158(52.7)$ \\
Five & $34(11.3)$ \\
Six & $6(2.0)$ \\
Seven & $1(0.3)$ \\
Total & $\mathbf{3 0 0}(\mathbf{1 0 0})$ \\
\hline
\end{tabular}

* Parenthes indicates percentage

About $4 \%$ patients were prescribed up to 2 drugs and the rest $96 \%$ patients were prescribed from 3 to 7 drugs. No single drug was found to be prescribed by generic names. In the present study, the percentage of encounters with an antibiotic and an injection prescribed were $6.67 \%$ and $3.33 \%$ respectively. Only 49(4.32\%) drugs out of 1134 drugs in 300 prescriptions were prescribed from the national EDL (Table II).

Table 2: Drugs prescribed from National EDL $(\mathrm{N}=1134)$

\begin{tabular}{lr}
\hline Drugs & Total Number of Drugs \\
\hline Included within EDL & $49(4.3)$ \\
Excluded from EDL & $1085(95.7)$ \\
Total & $\mathbf{1 1 3 4}(\mathbf{1 0 0 . 0})$ \\
\hline
\end{tabular}

*Parenthes indicates percentage

* EDL=Essential drug Ltd.

In the study, the most commonly prescribed essential and non-essential drugs were prednisolone (9.3\%) and pantoprazole $(73 \%)$ respectively. The three most commonly prescribed drugs which were included within or excluded from the national EDL are shown in Table 3. In the study, percentage of encounters with an antiulcerant and a NSAID prescribed were $97.0 \%$ and $97.3 \%$ respectively.

Table 3: Three most commonly Prescribed drugs included within or excluded from the National EDL $(\mathrm{N}=300)$

\begin{tabular}{lc}
\hline Drugs & Number of Prescriptions \\
\hline Included within EDL & \\
Prednisolone & $28(9.3)$ \\
Paracetamol & $13(4.3)$ \\
Tetanus toxoid & $03(1.0)$ \\
Excluded within EDL & \\
Pantoprazole & $219(73.0)$ \\
Calcium preparation & $202(67.3)$ \\
Ketoprofen & $130(43.3)$ \\
\hline
\end{tabular}

*Parenthes indicates percentage 
It was also seen that out of 300 prescriptions $202(67.3 \%)$ had at least one calcium preparation prescribed which was not included in national EDL.

Table 4: The overall findings for the WHO core prescribing Indicators

\begin{tabular}{lr}
\hline WHO core prescribing Indicators & Findings \\
\hline Prescribing indicators & \\
1. Average number of drugs per prescription & 3.8 \\
2. Percentage of drugs prescribed by generic name & 0.0 \\
3. Percentage of encounters with an antibiotic prescribed & 6.7 \\
4. Percentage of encounters with an injection prescribed & 3.3 \\
5. Percentage of drugs prescribed from national essential drug & 4.3 \\
list & \\
Some additional indices & \\
6. Percentage of encounters with an antiulcerant prescribed & 97.3 \\
7. Percentage of encounters with a NSAID prescribed & 97.0 \\
8. Percentage of encounters with a calcium preparation & 67.3 \\
prescribed & \\
\hline
\end{tabular}

\section{Discussion}

The study showed that the pattern of drug prescribing was not completely based on WHO criteria for rational use of drugs. The prescribing system was not at all evidence-based. With regard to the average number of drugs per prescription, the value found in the present study was 3.78. In similar studies conducted, the lower values found were 1.65 in Zimbabwe 92.7 in India ${ }^{10}$ and 2.91 in $\mathrm{Nepal}^{11}$. It also showed that more than $96 \%$ of the patients were given three or more drugs.

Since, WHO has recommended that average number of drugs per prescription should be 2.012 , so the results of the study reflect polypharmacy which may lead to adverse drug reactions, increase the risk of drug interactions, dispensing errors, medication errors, decrease adherence to drug regimens and unnecessary drug expenses. Use of generic names in prescription eliminates the chance of duplication of drug products and also reduces the cost of the patient ${ }^{13}$. The percentage of drugs prescribed by generic name was $0 \%$ in the study which is very much less than that reported in studies conducted in Cambodia ${ }^{14}(99.8 \%)$, $\operatorname{India}^{15}(73.4 \%)$ and $\mathrm{Nepal}^{16}(21.3 \%)$. The most common reasons for not prescribing generic name in Bangladesh may be tradition, low production of generic drugs in Bangladesh and currently, most of the pharmaceutical companies' divertive drug promotion technique. In the present study, the percentage of encounters with an antibiotic prescription was $6.67 \%$. According to WHO, $15-25 \%$ of antibiotics encountered is expectable in the countries where an infectious disease is more prevalent ${ }^{4-8}$. So this result indicates that the antimicrobial prescribing pattern was acceptable according to WHO guideline.

The WHO recommended target for injection exposure is $10 \%$ or less ${ }^{4}$. In this study, the percentage of prescriptions with an injection encountered was $3.33 \%$. So the observed proportion of injectable drugs prescribed is considered acceptable according to WHO recommendations. Minimum use of injections is preferred and this reduces the risk of infection through parenteral route and cost incurred in therapy ${ }^{17}$. In this study, the percentage of drugs prescribed from national EDL was only $4.32 \%$. The possible reason for this lower value could be the prescribers lacking the understanding the importance of essential drug concept. The low rate of prescribing from EDL of Bangladesh may be also contributed by excessive use of calcium preparations, antiulcerants (pantoprazole, esomeprazole) and NSAIDs (ketoprofen, naproxen) which are not enlisted in EDL of Bangladesh. So, that the higher percentage of non-essential medicines prescription in this study is responsible for inappropriate use of medicines.

In this study, pain is the most commonly experienced symptom among the patients. So the percentage of use of analgesics usually NSAIDs due to its less adverse effects was very high $(97 \%)$. The study also showed that antiulcer drugs were prescribed along with analgesics to reduce the gastric complications. So the percentage of encounters with an antiulcerent prescribed was $97.3 \%$. It also showed that out of 300 prescriptions $202(67.3 \%)$ had at least one calcium preparation prescribed which was not enlisted in EDL. The justification for this practice in most of the prescriptions is not clear.

\section{Conclusion}

Among the five steps of core prescribing indicators, only the percentage of encounters with an antibiotic and an injection prescribed was within the WHO recommendations. Otherwise, the mean number of drugs was very high, the percentage of prescribing by generic name was totally nil and percentage of drugs prescribed from national essential drug list was very low. So, educational interventions to improve prescribing for doctors at different levels may be urgently required.

\section{Acknowledgements}

We acknowledge the Principal of East-West Medical College and the Medical Director of the Hospital for administrative support surveyed for their cooperation.

\section{References}

1. Adiga MNS, Alwar MC, Pai MRSM, Adiga US. Pattern of antimicrobial agents use in hospital deliveries: A prospective comparative study. Online J Health Allied Scs 2009;8(4):10

2. World Health Organization (WHO). Introduction to drug utilization research. Oslo: 2003.

3. Brodie DC. Drug utilization review. Amer J Public Health 1972; 46: 103-109. 4. World Health Organization (WHO). International Network for Rational Use of Drugs and World Health Organization. How to investigate drug use in health facilities: Selected drug use indicators 1993. EDM Research Series No. 7 [WHO/DAP/93.1].

5. Hogerzeil HV. Promoting rational prescribing: an international perspective. Br J Clin Pharmacol 1995; 39: 1-6.

6. Gaud RS, Jain DK, Kaskhedikar SG, Chaturvedi SC. Critical evaluation of present prescribing pattern. Indian J Hosp Pharm 1989; 26: 70-72.

7. Alam MM, Parveen F, Ara F, Iqbal MJU, Saha RR. Prescribing trends in the out patient department in a tertiary hospital in Bangladesh. Bangladesh Medical Journal 2011; 40 (2): 8-12.

8. Bhartiy SS, Shinde M, Nandeshwar S, Tiwari SC. Pattern of prescribing practices in the Madhya Pradesh, India. Kathmandu Univ Med J 2008; 6: 55-59. 
9. Lessing C, Trap B. Zimbabwe Essential Drugs Action Programme (ZEDAP) 1995. Ministry of Health and Child Welfare Directorate of Pharmacy.

10. Mhetre NA, Bodhankar SL, Pandit VA, Zambare GN. Study of pattern of drug usage in an urban area. Indian J Pharmacol 2003; 35: 316-317.

11. Alam K, Mishra P, Prabhu M, Shankar PR, Palaian S, Bhandari RB, et al. A study on rational drug prescribing and dispensing in outpatients in a tertiary care teaching hospital of Western Nepal. Kathmandu Univ Med J 2006; 4: 436-443.

12. Sharif SI, Al-Shaqra M, Hajjar H, Shamout A, Wess L. Patterns of Drug Prescribing in a Hospital in Dubai, United Arab Emirates. LIJ, AOP 2007; 070928: 10-12.

13. Acurcio FA, Perini E, Magalhaes SM, Terceiro LG, Vieira Filho JM,
Coutinho KE et al. Analysis of medical prescriptions dispensed at health centers in Belo Horizonte, Minas Gerais, Brazil. Cad Saude Publica 2004; 20: 72-79.

14. Chareonkul C, Khun VL, Boonshuyar C. Rational drug use in Cambodia: study of three pilot health centers in Kampong Thom Province. Southeast Asian J Trop Med Public Health 2002; 33: 418-424.

15. Karande S, Sankhe P, Kulkarni M. Patterns of prescription and drug dispensing. Indian J Pediatr 2005; 72: 117-121.

16. Shankar PR, Pranab KS, Upadhyay DK, Dubey AK, Subish P. Drug Utilization among Surgical Outpatients. TMJ 2006; 56: 230-234.

17. Ghimire S, Nepal S, Bhandari S, Nepal P, Palaian S. A prospective surveillance of drug prescribing and dispensing in a teaching hospital in Western Nepal. J Pak Med Assoc 2009; 59: 726-731. 